\title{
Counter-rotation in relativistic magnetohydrodynamic jets
}

\author{
V. Cayatte $^{1}$ \\ N. Vlahakis ${ }^{2}$ \\ T. Matsakos ${ }^{3,4}$ \\ J.J.G. Lima ${ }^{5,6}$ \\ K. Tsinganos ${ }^{2,7}$ \\ C. Sauty ${ }^{1}$ \\ veronique.cayatte@obspm.fr
}

Received __; accepted _

\footnotetext{
${ }^{1}$ Laboratoire Univers et Théories, Observatoire de Paris, UMR 8102 du CNRS, Université Paris Diderot, F-92190 Meudon, France

${ }^{2}$ Department of Astrophysics, Astronomy and Mechanics, Faculty of Physics, University of Athens, 15784 Zografos, Athens, Greece

${ }^{3}$ Department of Astronomy \& Astrophysics, The University of Chicago, Chicago, IL 60637, USA

${ }^{4}$ LERMA, Observatoire de Paris, Université Pierre et Marie Curie, Ecole Normale Supérieure, Université Cergy-Pontoise, CNRS, France

${ }^{5}$ Centro de Astrofísica, Universidade do Porto, Rua das Estrelas, 4150-762 Porto, Portugal

${ }^{6}$ Departamento de Física e Astronomia, Faculdade de Ciências, Universidade Porto, Rua do Campo Alegre, 687, 4169-007 Porto, Portugal

${ }^{7}$ National Observatory of Athens, Lofos Nymphon, Thission 11810, Athens, Greece
} 


\begin{abstract}
Young stellar object observations suggest that some jets rotate in the opposite direction with respect to their disk. In a recent study, Sauty et al. (2012) have shown that this does not contradict the magnetocentrifugal mechanism that is believed to launch such outflows. Signatures of motions transverse to the jet axis and in opposite directions have recently been measured in M87 (Mever et al., 2013). One possible interpretation of this motion is the one of counter rotating knots. Here, we extend our previous analytical derivation of counter-rotation to relativistic jets, demonstrating that counter-rotation can indeed take place under rather general conditions. We show that both the magnetic field and a non-negligible enthalpy are necessary at the origin of counter-rotating outflows, and that the effect is associated with a transfer of energy flux from the matter to the electromagnetic field. This can be realized in three cases : if a decreasing enthalpy causes an increase of the Poynting flux, if the flow decelerates, or, if strong gradients of the magnetic field are present. An illustration of the involved mechanism is given by an example of relativistic MHD jet simulation.
\end{abstract}

Subject headings: galaxies: active — galaxies: jets — magnetohydrodynamics — relativistic processes — quasars: supermassive black holes 


\section{Introduction}

In a previous Letter (Sauty et al. 2012) we have established that counter rotation in jets from young stars could be a natural consequence of the MHD equations ruling the plasma. We have shown that deceleration of the jet or shocks can induce counter rotation. We have verified it analytically and numerically. In young stars, it is possible to measure observationally the rotation speed of the jet. Counter-rotation has been observed in some cases but remains under debate. However, in the light of our criterion, it is clear that it does not contradict the magnetorotational launching of the jet.

In the context of relativistic outflows such as AGN jets, the criterion, if it can be extended, may induce crucial observational consequences. Those jets are well known to be magnetically launched as well. Recent measurements of the polarization and the VLBI Faraday rotation (e.g. Mahmud et al. 2013; Algaba et al. 2013) have confirmed the helicoidal nature of the jet magnetic field which supports the idea of magnetic launching. Faraday rotation measures provide a direct evidence of the magnetic field structure within the jet (Gabuzda 2003) and allow to confront observations with simulations (Broderick \& McKinney 2010). Besides, Meyer et al. (2013) have measured transverse proper motions of knots in the jet of M87. They infer that knots A and $\mathrm{C}$ seem to have opposite velocities transverse to the jet which they interpret as counter rotating shocks. They claim that this is consistent with the model of quad relativistic MHD shocks of Nakamura, Garofalo, \& Meier (2010). This model was used to interpret the helical magnetic structure inferred from polarization measurements for the same knots by Algaba et al. (2013). It is worth to note that these observations are rather difficult and need a very long time survey.

Komissarov et al. (2009) have shown numerically that counter-rotation appears in their simulations (hot jet of model B2H) as the result of transfer of angular momentum from the 
fluid to the magnetic component. Nakamura, Garofalo, \& Meier (2010) have also shown that the complex structure of the quad relativistic MHD shock model exhibits a reverse shock that flows upstream, rotating in the direction opposite to the forward shock.

We show in this Letter a straightforward extension in the relativistic regime of our criterion for counter rotation in young stars which can also be interpreted in terms of the flow energetics. This criterion applies to shock models as well as simulations where the angular momentum and isorotation frequency are conserved. This does not necessarily means it applies to quad shock models. The compatibility with the Riemann problem solution still needs to be checked. However, the present criterion involved a simpler geometry and does not rely on the presence of kink instabilities or precession of the jet axis.

Then, even though rotation measurements in relativistic sources are still out of reach, counter-rotation may have strong observational signatures related to the magnetic field structures. In fact, precise measurements of the magnetic field gradients would be needed to get constraints on the jet dynamics and rotation.

\section{Steady axisymmetric relativistic MHD outflows}

\subsection{Integrals of motion in Kerr metrics}

Under the assumption of steady-state and axisymmetry the equations of general relativistic magnetohydrodynamics can be partially integrated to yield several field/streamline constants (e.g., Beskin 2010), including the magnetic field angular velocity

$$
\Omega=\omega+\frac{h}{\varpi}\left(V_{\varphi}-\frac{V_{\mathrm{p}}}{B_{\mathrm{p}}} B_{\varphi}\right),
$$

the total angular momentum flux to mass flux ratio

$$
L=\xi \gamma \varpi V_{\varphi}-\frac{\varpi B_{\varphi} B_{\mathrm{p}}}{4 \pi \gamma \rho_{0} V_{\mathrm{p}}},
$$


and the total energy flux to mass flux ratio

$$
\mu c^{2}=h \xi \gamma c^{2}+\xi \gamma \omega \varpi V_{\varphi}-\frac{\varpi \Omega B_{\varphi} B_{\mathrm{p}}}{4 \pi \gamma \rho_{0} V_{\mathrm{p}}} .
$$

Here $h$ is the lapse function, $\varpi\left(=\sqrt{g_{\varphi \varphi}}\right)$ is the cylindrical radius in Kerr geometry, $\omega$ is the angular velocity of zero angular momentum observers (ZAMO), $B_{\mathrm{p}}, B_{\varphi}, V_{\mathrm{p}}, V_{\varphi}$ denote poloidal and toroidal components of the magnetic field and bulk flow speed as seen by ZAMO, $\gamma$ is the bulk Lorentz factor, and $\left(\rho_{0}, \xi c^{2}\right)$ are the mass density and specific enthalpy as measured in the frame comoving with the outflow.

The system of the previous three equations can be solved for $\left(\gamma, B_{\varphi}, \gamma V_{\varphi}\right)$ as it is usually done. Equivalently we may use instead $\left(h \gamma \xi c^{2}, B_{\varphi}, \gamma \xi V_{\varphi}\right)$. This gives the following expressions in the observer's frame for the matter part of the energy flux to mass flux ratio

$$
h \gamma \xi c^{2}=\frac{M^{2}\left(\mu c^{2}-L \omega\right)-h^{2}\left(\mu c^{2}-L \Omega\right)}{M^{2}-h^{2}+\varpi^{2}(\Omega-\omega)^{2} / c^{2}},
$$

and the toroidal proper component of the proper specific momentum

$$
\xi \gamma V_{\varphi}=\frac{L}{\varpi} \frac{M^{2}-\varpi^{2}(\Omega-\omega)\left(\mu c^{2}-L \Omega\right) / L c^{2}}{M^{2}-h^{2}+\varpi^{2}(\Omega-\omega)^{2} / c^{2}}
$$

in terms of the square of the "Alfvénic" Mach number

$$
M^{2}=4 \pi \xi \rho_{0} h^{2} \gamma^{2} V_{\mathrm{p}}^{2} / B_{\mathrm{p}}^{2}
$$

The numerators and denominators of Eqs. (44)-(5) vanish at the Alfvén surface (subscript $\star$ ) where the values of $M^{2}, \varpi$ are given by

$$
\frac{M_{\star}^{2}}{h_{\star}^{2}}=\frac{\mu c^{2}-L \Omega}{\mu c^{2}-L \omega_{\star}}, \quad \varpi_{\star}^{2}=\frac{L h_{\star}^{2} c^{2}}{\left(\Omega-\omega_{\star}\right)\left(\mu c^{2}-L \omega_{\star}\right)} .
$$

\subsection{Generic criterion in Kerr metrics for counter-rotation}

Assuming that the flow remains everywhere super-Alfvénic after crossing the corresponding critical surface, the denominator of Eq. (5) is always positive. The sign of 
the toroidal velocity is then given by the sign of the numerator, and thus we get that it is negative if,

$$
M^{2}<\varpi^{2}(\Omega-\omega) \frac{\mu c^{2}-L \Omega}{L c^{2}}
$$

Along a given flux tube, we can write the magnetic and mass flux conservation as

$$
B_{\mathrm{p}} \delta S=B_{\star} \delta S_{\star}=\text { constant }
$$

and

$$
h \gamma \rho_{0} V_{\mathrm{p}} \delta S=h_{\star} \gamma_{\star} \rho_{0 \star} V_{\star} \delta S_{\star}=\text { constant } .
$$

Note that $\delta S$ is the surface element perpendicular to the poloidal velocity.

Using the definition of $M^{2}$ and the last two conservation laws following the procedure of the previous paper, we show that the condition (8) is equivalent to,

$$
\frac{4 \pi \rho_{0 \star} h_{\star} \gamma_{\star} V_{\star} \frac{\delta S_{\star}}{\delta S}}{B_{\star}^{2} \frac{\delta S_{\star}^{2}}{\delta S^{2}}} h \xi \gamma V_{\mathrm{p}}<\varpi^{2}(\Omega-\omega) \frac{\mu c^{2}-L \Omega}{L c^{2}} .
$$

Using Eqs. (7) and (6) at the Alfvén surface, the above expression simplifies further,

$$
\frac{h \xi \gamma V_{\mathrm{p}} \delta S}{h_{\star} \xi_{\star} \gamma_{\star} V_{\star} \delta S_{\star}}<\frac{\varpi^{2}(\Omega-\omega)}{\varpi_{\star}^{2}\left(\Omega-\omega_{\star}\right)} .
$$

Thus, the last inequality determines if the toroidal velocity is negative.

If the flow remains super-Alfvénic, reversal of the rotation takes place when the proper velocity times the enthalpy drops below some threshold value. The flow can decelerate or lose enthalpy either because it expands and this causes adiabatic cooling, or because its kinetic energy drops due to some other mechanism such as radiative losses. Note that we used in Eq. (11) energy conservation. However the criterion in Eq. (12) remains valid if the energy downstream, $\mu_{\text {local }}$, is lower than the energy at the Alfvén surface because in that case,

$$
\varpi^{2}(\Omega-\omega) \frac{\mu_{\mathrm{local}} c^{2}-L \Omega}{L c^{2}}<\varpi^{2}(\Omega-\omega) \frac{\mu_{\star} c^{2}-L \Omega}{L c^{2}}
$$


Rotation reverses also if the threshold value increases and gets larger than the jet poloidal velocity.

In the sub-Alfvénic regime, close to the Black Hole, counter rotation may occur as well though the reverse inequality holds. Above some threshold value of the energy, the rotation velocity reverses. In other words very close to the central object, counter-rotation may occur if there is a steep acceleration followed by a mild deceleration before the Alfvén point. This is the case of the solution presented for RY Tau in Sauty et al. (2011). We have explored a generalization of this solution in Kerr metrics and we suspect that rotation also reverses in the sub-Alfvénic regime there.

In the far asymptotic regime where the flow is super-Aflvénic and special relativistic $(h \simeq 1$ and $\omega \ll \Omega)$, the flow rotation reverses if,

$$
\frac{\xi \gamma V_{\mathrm{p}} \delta S}{\xi_{\star} \gamma_{\star} V_{\star} \delta S_{\star}}<\frac{\varpi^{2}}{\varpi_{\star}^{2}} .
$$

If the flow is sufficently smooth with $\delta S \propto \varpi^{2}$ and with ultrarelativistic velocities $\left(V_{\mathrm{p}}\right.$ and $\left.V_{\star} \simeq c\right)$, the inequality simplifies to,

$$
\xi \gamma<\xi_{\star} \gamma_{\star}
$$

For an accelerated flow, this inequality implies that $\xi=1+w / c^{2}$, where $w$ is the part of the specific enthalpy without the rest-mass density, should decrease after the Alfven surface.

\subsection{Criterion in terms of the flow energetics}

In the following section, we restrict our discussion to a flow with constant energy flux. The hydrodynamic and electromagnetic contributions in the angular 
momentum and energy fluxes can be easily identified by inspecting Eqs. (2) and (3). We can write $L=L_{\mathrm{HYD}}+L_{\mathrm{MAG}}$ and $\mu=\mu_{\mathrm{HYD}}+\mu_{\mathrm{MAG}}$, where $L_{\mathrm{HYD}}=\xi \gamma \varpi V_{\varphi}$, $L_{\mathrm{MAG}}=-\varpi B_{\varphi} B_{\mathrm{p}} /\left(4 \pi \gamma \rho_{0} V_{\mathrm{p}}\right), \mu_{\mathrm{HYD}}=h \xi \gamma+\xi \gamma \omega \varpi V_{\varphi} / c^{2}, \mu_{\mathrm{MAG}}=L_{\mathrm{MAG}} \Omega / c^{2}$.

Near the regime where the toroidal speed changes sign from positive to negative, $L_{\mathrm{HYD}}$ is a decreasing function of distance, and since $L$ is constant, the electromagnetic part $L_{\mathrm{MAG}}$ should be an increasing function. The same should hold for the corresponding parts of the energy to mass flux ratio: The electromagnetic part $\mu_{\mathrm{MAG}}$ is proportional to $L_{\mathrm{MAG}}$ and thus increses with distance, while the hydromagnetic part $\mu_{\mathrm{HYD}}=\mu-\mu_{\mathrm{MAG}}$ decreases. As a result, at least in the region where $V_{\varphi}$ changes sign, energy should be transferred from the matter to the electromagnetic part of the outflow. This can be realized in a decelerating cold flow, or in an accelerating hot flow in which part of the enthalpy is transferred to the electromagnetic field. The last case has been seen in the simulations of the following section, and also in one of the simulations of Komissarov et al. (2009). Section 5.5 of that paper includes a connection of the possible counter-rotation of the jet with the poloidal field/streamline shape (which in turn is related to the magnetic energy density and the Poynting flux through the bunching function), as well as a discussion on why previous self-similar solutions of the relativistic MHD hot outflow problem (Vlahakis \& Königl 2003) do not show counter-rotating jets.

Another connection of counter-rotation with the flow energetics follows from a combination of Eqs. (21) and (3)

$$
\frac{\xi \gamma \varpi \Omega V_{\varphi}}{\mu c^{2}}=\frac{L \Omega}{\mu c^{2}}-\frac{\sigma}{1+\sigma},
$$

where $\sigma=\mu_{\mathrm{MAG}} / \mu_{\mathrm{HYD}}$ is the magnetization function. Negative $V_{\varphi}$ requires a sufficiently magnetized flow such that $\sigma>L \Omega /\left(\mu c^{2}-L \Omega\right)$. This in turn implies that $L \Omega$ must be significantly smaller than $\mu c^{2}$, contrary to outflows that are strongly Poynting-dominated near their origin (in which $\mu c^{2} \approx \mu_{\mathrm{MAG}} c^{2}=L_{\mathrm{MAG}} \Omega \approx L \Omega$ ). Consequently, the enthalpy 
part of the energy flux is nonnegligible near the origin of counter-rotating outflows.

\section{Counter-rotation in numerical simulations}

\subsection{Numerical setup}

In order to illustrate the above demonstration, we perform a numerical simulation of a jet using the relativistic MHD framework of the PLUTO code (Mignone et al. 2007). We assume axisymmetry, an ideal equation of state with polytropic index $4 / 3$, and focus in the superfast region of the outflow. The initial conditions provide an equilibrium along the radial direction, but not in the vertical one. We choose for the vertical magnetic field a field that decreases along the jet as a power law related to the natural transverse expansion of the jet. At a given altitude, close to the axis the vertical magnetic field is nearly constant and decreases transversely as $1 / \varpi^{2}$ such that the total magnetic flux does not diverge,

$$
B_{z}=\frac{2 c_{1} c_{2}}{z^{2 / b}+c_{2} \varpi^{2}}
$$

Such a choice, together with a radial magnetic field

$$
B_{\varpi}=\frac{\varpi}{b z} B_{z}
$$

which is consistent with parabolic poloidal field lines $z \propto \varpi^{b}$ (Heyvaerts \& Norman 1989), satisfies the divergence free condition.

The toroidal magnetic field is deduced from the vertical magnetic field such that the poloidal current intensity becomes constant far from the axis. The toroidal velocity field is taken to be linear close to the axis consistently with a jet from a solid body rotator and decreases at large distances as the inverse of the distance, similarly to the toroidal magnetic field,

$$
B_{\varphi}=-\varpi B_{z}, \quad V_{\varphi}=V_{\varphi ; 0} \frac{c_{2} \varpi}{z^{2 / b}+c_{2} \varpi^{2}}
$$



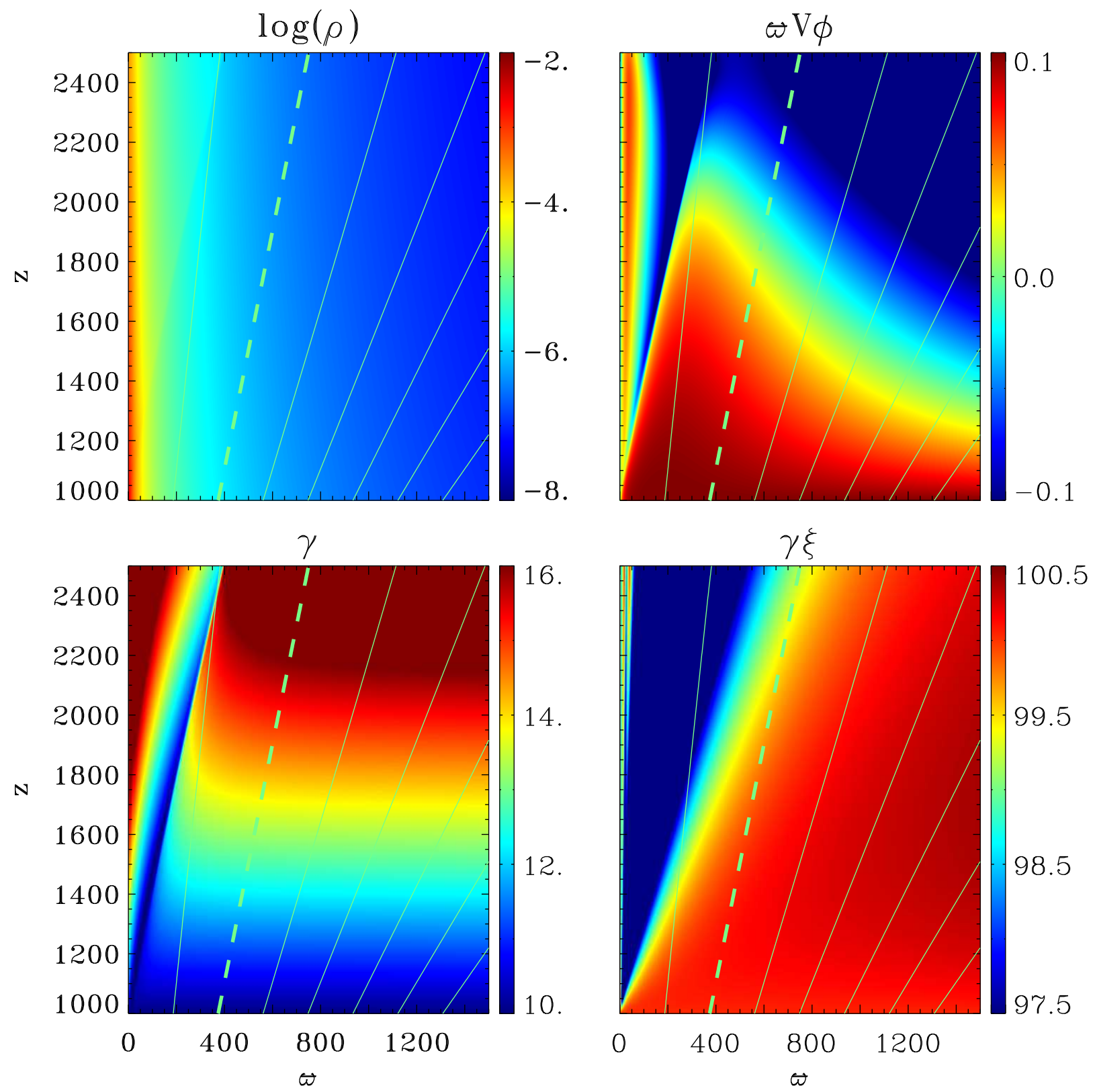

Fig. 1.- Maps of the density (top left), Lorentz factor (bottom left), toroidal velocity multiplied by the radius (top right), relativistic enthalpy (bottom right), together with the magnetic field lines. Whenever the values of each quantity are outside the plotted range, the colors saturate to the corresponding $\min / \max$ value. 

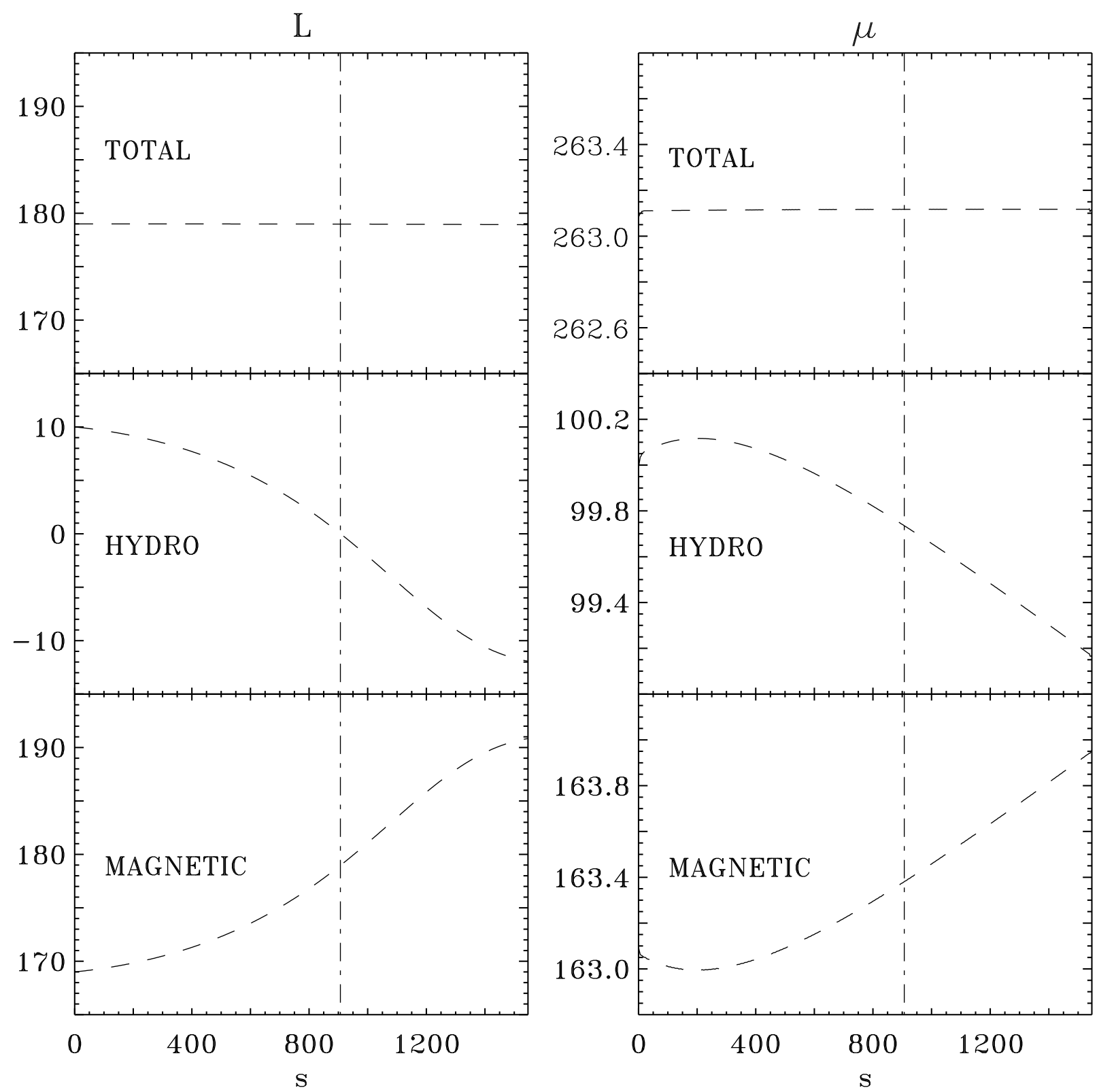

Fig. 2.- The profiles of $L$ (left) and $\mu$ (right) along the dashed field line indicated in Fig. [1 The total values of the integrals are broken to the hydro and magnetic components. The vertical dot-dashed line shows the location at which counter-rotation starts. 
To start as close as possible from a steady state, we choose the poloidal velocity parallel to the initial poloidal magnetic field (equivalently the toroidal component of the electric field vanishes). Using the definition of the Lorentz factor we get,

$$
V_{\varpi}=\sqrt{\frac{1-1 / \gamma^{2}-V_{\varphi}^{2}}{1+B_{z}^{2} / B_{\varpi}^{2}}}, \quad V_{z}=\frac{B_{z}}{B_{\varpi}} V_{\varpi} .
$$

The equilibrium is statisfied only in the radial direction. We assume a $4 / 3$ polytrope and obtain the initial density and pressure distributions,

$$
\rho_{0}=\frac{8 c_{1}^{2} c_{2}^{2} \varpi^{2}\left(z^{2 / b}-c_{2} \gamma^{2}\right)}{\gamma^{4} \xi\left(z^{2 / b}+c_{2} \varpi^{2}\right)^{3} V_{\varphi}^{2}}, \quad P=\frac{\xi-1}{4} \rho_{0},
$$

where $\gamma=10$ and $\xi=10$ are the initially uniform Lorentz factor and specific enthalpy. We choose $c_{1}=10, c_{2}=99.5, b=1.5$, and $V_{\varphi ; 0}=0.1$ for the other constants. The value of $c_{1}$ provides the strength of the magnetic field, whereas $c_{2}$ sets the radial distance beyond which $B_{z}$ and $V_{\varphi}$ become pure power laws of $\varpi$. The exponent $b$ determines the shape of the poloidal field/streamlines.

The size of the box is $\varpi \times z=[0,1] \cdot 10^{3} \times[1,2.5] \cdot 10^{3}$ and is resolved by 512 zones in each direction. A stretched grid of 256 zones extends the radial direction up to $\varpi=10000$ in order to minimize boundary effects. The speed of light is set to unity, the length is normalized in units of the light cylinder, and the magnetic fields have absorbed the factor $1 / \sqrt{4 \pi}$. Axisymmetric conditions are applied on the left boundary of the computational domain, outflow on the top and right edges 1 and the initial conditions are kept fixed on the lower boundary. We carry out the simulation up to $t=50000$, but we note that a steady-state is reached within a fraction of this time.

\footnotetext{
${ }^{1}$ We have verified that the results are robust no matter what boundary conditions are applied on the right side of the box.
} 


\subsection{Results}

During the first steps of the simulation the flow reaches a steady-state as the information from the bottom boundary propagates upwards. An oblique shock forms close to the axis, a feature which is common to radially self-similar outflow simulations (see Matsakos et al. 2008 for a discussion). Even though counter-rotation effects take place due to the shock (see Fendt 2011 for a discussion of the mechanism), in this paper we focus farther away from the axis where the flow is smooth. Figure 1 displays the final configuration of the jet at $t=50000$. The flow is accelerated along the field lines as it can be seen by the gradual increase of $\gamma$. However, the hydrodynamic part of the energy to mass flux ratio, $\xi \gamma$, decreases and hence a drop in $V_{\varphi}$ is anticipated as shown in Sect. 2.3. Indeed, the toroidal velocity decreases and in fact it becomes negative above a certain height, beyond which the jet counter-rotates.

In more detail, Fig. 2 shows that the total angular momentum to mass flux ratio $(L)$ and the total energy to mass flux ratio $(\mu)$ are constant along the field/streamlines as expected in axisymmetric steady states. However, energy is transfered from the matter to the electromagnetic field ( $\mu_{\mathrm{HYD}}$ decreases and $\mu_{\mathrm{MAG}}$ increases). In addition, since the magnetic terms in the energy and angular momentum flux are proportional, the term $L_{\mathrm{HYD}}$ is also converted to $L_{\mathrm{MAG}}$ in order for the angular momentum flux to remain constant.

Here, we note that the details of the final configuration of the setup depend weakly on the resolution as well as the location of the outer boundary. However, the counter-rotation effect we have described is a robust feature that appears in all variations of our simulated numerical model. Specifically, the morphology of the integrals and their components have the same profile in all cases examined, following the prediction of the analytical demonstration. 


\section{Conclusion}

In Sauty et al. (2012), as well as in this Letter we have shown that counter-rotation is a signature of the magnetization of a jet, both in the classical and the relativistic regimes. Counter-rotation is possible in the following three cases, the first two ones refer to the geometry of the flow while the third one is related to the energetics of the jet,

1. Gradients of the magnetic field are associated with a compression of the flow and a sufficiently small $\delta S$ such that Eq. (13) is satisfied.

2. In a smooth flow in which $\delta S \propto \varpi^{2}$, a decrease of $\mu_{\mathrm{HYD}}$ may lead to the inequality (13). This can happen if the flow is decelerated.

3. In an ultrarelativistic accelerated or constant flow with $\delta S \propto \varpi^{2}$, part of the decreasing enthalpy flux could be transfered to the electromagnetic field, see Sect. 2.3. Then the hydrodynamic part of the energy to mass flux ratio $\mu_{\mathrm{HYD}}$ should be smaller than its value at the Alfvén surface to obtain counter-rotation.

In all cases, the role of the magnetic field is critical because it provides the agent to absorb the excess of matter angular momentum. Therefore, counter-rotation is only possible in MHD outflows. As explained at the end of Sect. 2.3 the thermal content in these flows is also important at least near their origin.

The first case may be obtained if there are surface instabilities at the edge of the jet. In this case although the jet expands the flux tubes may squeeze. The changes in the size of the flux tube can induce counter rotation.

The second case may occur in FRI jets. As a matter of fact, FRI radio galaxies usually exhibit ultra relativistic jets on the parsec scale while the kilo parsec jet is mildly or not relativistic. It is usually interpreted as a strong deceleration of the moving plasma (see 
Meliani et al. 2010 and references therein). This strong deceleration may correspond to the second case where we would observe counter-rotation. This is comparable to the non relativistic case studied in (Sauty et al. 2012). Conversely, if the kilo parsec scale outflow were to be an outer component slower than the inner relativistic spine jet that we see on the parsec scale, provided that both components are accelerated, then it is likely that the jet rotation sense would be the same on all scales. Measuring the rotation of the flow would thus allow to distinguish between a single component decelerating outflow which changes sense of rotation and a two component accelerating jet which does not changes rotation.

The third case corresponds to a decrease of the total enthalpy budget at large distances. In such ultrarelativistic outflows, the transformation of thermal energy flux (enthalpy) to Poynting energy flux would induce counter-rotation and a strong increase in the toroidal magnetic field, i.e. matter energy is converted into magnetic energy. The change of sign of the rotational speed would induce an increase of the Poynting flux and of the magnetic component of the total angular momentum. As a byproduct, the toroidal magnetic field would increase as well, creating strong gradients of the toroidal magnetic field in the poloidal direction. At present, the magnetic field direction is measured via the change of Faraday rotation across the jet (Mahmud et al. 2013). A complex helicoidal magnetic structure in the knots $\mathrm{A}$ and $\mathrm{C}$ of M87 jet has already been associated with possible counter rotation as we mentioned in the Introduction (Algaba et al., 2013, see also Meyer et al., 2013 and Nakamura, Garofalo, \& Meier, 2010).

Altogether then, also in relativistic outflows counter-rotation does not contradict at all magnetic launching. Instead, it provides another piece of evidence for the essential role played by the magnetic field in the flow. It is important to measure the sense of rotation along the jet, because we may get, among others, an evidence for the energetic and angular momentum interchanges in the jet between its fluid and magnetic parts. 
The authors thank an anonymous referee for her/his valuable comments which help us to improve the manuscript. This work has been supported by the project "Jets in young stellar objects: what do simulations tell us?" funded under a 2012 Égide/FranceFCT/Portugal bilateral cooperation Pessoa Programme and by the Scientific Council of Paris Observatory (PTV program). NV acknowledges the hospitality and support of the Laboratoire Univers et Théories, Observatoire de Paris, during his visit supported by the Scientific Council of Paris Observatory (PTV program). TM was supported in part by NASA ATP grant NNX13AH56G. 


\section{REFERENCES}

Algaba, J. C., Asada, K., \& Nakamura, M. 2013, preprint arXiv:1308.5429

Beskin, V. S. 2010, MHD Flows in Compact Astrophysical Objects: Accretion, Winds and Jets (Springer-Verlag Berlin Heidelberg)

Broderick, A. E., \& McKinney, J. C. 2010, ApJ, 725, 750

Fendt, C. 2011, ApJ, 737, 43

Gabuzda, D. C. 2003, NewAR, 47, 599

Heyvaerts, J., Norman, C. A. 1989, ApJ, 347, 1055

Komissarov, S. S., Vlahakis, N., Königl, A., \& Barkov, M. V. 2009, MNRAS, 394, 1182

Mahmud, M., Coughlan, C. P., Murphy, E., Gabuzda, D. C., \& Hallahan, D. R. 2013, MNRAS, 431, 695

Matsakos, T., Tsinganos, K., Vlahakis, N., et al. 2008, A\&A, 477, 521

Meliani, Z., Sauty, C., Tsinganos, K., Trussoni, E., \& Cayatte, V. 2010, A\&A, 521, A67

Meyer E. T., Sparks W. B., Biretta J. A., Anderson J., Sohn S. T., van der Marel R. P., Norman C., Nakamura M. 2013, ApJ, 774, L21

Mignone, A., Bodo, G., Massaglia, S., et al. 2007, ApJS, 170, 228

Nakamura M., Garofalo D., Meier D. L. 2010, ApJ, 721, 1783

Sauty, C., Meliani, Z., Lima, J. J. G., Tsinganos, K., Cayatte, V., \& Globus, N. 2011, A\&A, 533, A46

Sauty, C., Cayatte, V., Lima, J. J. G., Matsakos, T., \& Tsinganos, K. 2012, ApJ, 759, L1 
Vlahakis, N., \& Königl, A. 2003, ApJ, 596, 1080 